\title{
Augmented Reality in Drug Package: a Promising Strategy against Non-adherence in the Treatment of Tuberculosis
}

\author{
Dayse E. de Oliveira, Marcus V. N. de Souza, ${ }^{*}$ and Wanise B. G. Barroso \\ Fundação Oswaldo Cruz (Fiocruz), Instituto de Tecnologia em Fármacos (Farmanguinhos), \\ Rua Sizenando Nabuco, 100, Manguinhos, 21041-250 Rio de Janeiro, Brazil
}

Email: mvndesouza@gmail.com (M. V. N. S.)

\begin{abstract}
Augmented reality is a technology-based on an interaction between the virtual and real-world, mixing them and expanding their possibilities. This technology is currently used in the most varied areas of human knowledge. The use of augmented reality in drug package has excellent potential in several diseases and could be an indispensable tool, especially in diseases with low medication adherence. In this context, its application in tuberculosis treatment could be of great help, as this treatment presents two major issues: the long-term treatment time (six months) and its severe side effects resulting in its non-adherence to the treatment. In this article, we aim to address the problems related to tuberculosis treatment causing non-adherence by using augmented reality. Using a smartphone in the medicine packaging could gain valuable information making patients be aware of following the treatment until the end.
\end{abstract}

Keywords augmented reality, tuberculosis, non-adherence treatment

\section{Introduction}

Tuberculosis (TB) is a disease with reports since the beginning of human civilization. This disease is still responsible for a large number of deaths around the world of people with different ages and social classes, especially the poorest. ${ }^{[1-3]}$ According to World Health Organization, an estimated 10 million people were affected by tuberculosis worldwide and a total of 1.5 million people died in $2018 .^{[4]}$ Despite being a disease constantly present in human history, its etiological agent was only identified in 1882 by the German Heinrich Hermann Robert Koch (1843-1910). ${ }^{[5,6]}$ Such agent is a bacteria known as Mycobacterium tuberculosis transmitted through the air. In the following century, we have significant advances in the treatment of various diseases. In the case of tuberculosis, with the identification of the Mycobacterium tuberculosis, several chemical compounds of synthetic and natural origin have been tested. After 64 years from Robert Kock's discovery, we had the first drug approved for the treatment of tuberculosis, a natural product named streptomycin (Figure 1). ${ }^{[7]}$ The natural product is extracted from the soil of Streptomyces griseus developed for tuberculosis by Selman Abraham Waksman (1888-1973) and his co-workers between 1943-1946. After the discovery of streptomycin in the 1940-1970 period, several natural and synthetic drugs were developed and used successfully in the treatment of tuberculosis. ${ }^{[8]}$ It is essential to highlight that virtually all current tuberculosis treatment comes from the drugs discovered in this period. This fact shows that the development of new medications is urgent and necessary. Tuberculosis treatment presents two major issues: long-term treatment time (six months) and its severe side effects resulting in its non-adherence to treatment and, consequently, the emergence of drug-resistant strains. There are currently several kinds of drug-resistant tuberculosis being a relevant issue in the treatment and cure of tuberculosis. Considering these issues, the objective of this work is to propose new strategies to combat non-adherence to tuberculosis treatment based on augmented reality. Augmented reality is a technology-based on an interaction between the virtual and real-world, mixing them and expanding their possibilities. In this paper, augmented reality could be an excellent tool in combating non-adherence to the medications against tuberculosis. The use of a smartphone in the medication packaging could obtain valuable information making the patient be aware of following the treatment until the end.

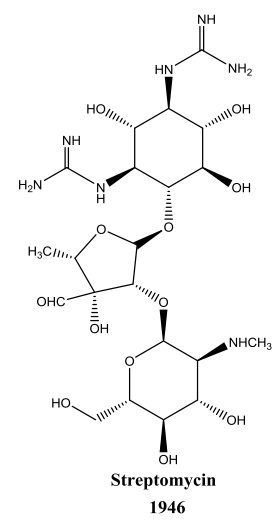

Figure 1 The structure of Streptomycin.

\section{Resurgence of Tuberculosis}

When there were several anti-tuberculosis drugs on the market after the 1970s, it was believed that tuberculosis would finally be eradicated. However, a disease emerged known as AIDS (Acquired Immune Deficiency Syndrome) caused by a retrovirus (RNA genome) named the Human Immunodeficiency Virus (HIV) in the 1980s. This contagious disease is capable of compromising the immunological system, being an important contributor to the resurgence of tuberculosis, which is the most common opportunistic infection and cause of death in AIDS, killing one every three patients. ${ }^{[11]}$ In the years that followed, other factors were responsible for the resurgence of 
tuberculosis, such as war, immigration, poverty, and the lack of new drugs. Nowadays, another major problem in tuberculosis treatment and also responsible for resurgence of tuberculosis is resistant strains. Due to all these problems, the World Health Organization declared tuberculosis was a global health emergency in 1993.

\section{Tuberculosis Treatment}

The current treatment against tuberculosis is divided into the first and second-line treatments. In the first-line, the essential drugs are isoniazid, rifampicin, pyrazinamide and ethambutol, presenting different mechanisms of action and used in combination. This strategy is necessary for an effective treatment avoiding the emergence of multiple drug-resistant organisms. $^{[9]}$ The standard drug-susceptible tuberculosis treatment is the combination of isoniazid, rifampin, pyrazinamide, and ethambutol for two months, followed by isoniazid and rifampin for four months (Figure 2). If followed as prescribed, this treatment is cheap and very effective in more than $95 \%$ of cases. It is important to emphasize that an essential strategy against abandonment in the treatment of tuberculosis is the use of fixed-dose combination (FDC) defined as a formulation of two or more substances biologically active, combined in a single drug, available in specific fixed doses. These four tuberculosis-drugs were developed in a single pill, as well as isoniazid plus rifampin existing other FDCs for tuberculosis treatment. Besides being effective against abandonment in the treatment of tuberculosis, this strategy has several advantages including a lower cost of treatment, better storage, distribution and shipping, reduction of drug-resistance, medication errors, dosages adjustment according to patients' needs, and better patient compliance. ${ }^{[10]}$

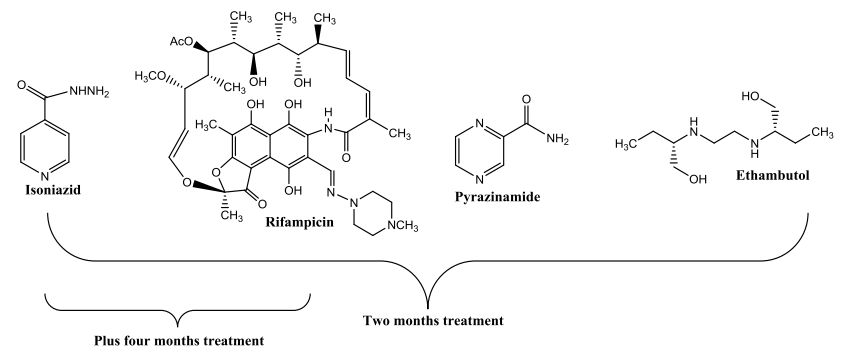

Figure 2 Standard drug-susceptible tuberculosis treatment.

\section{Second-line Tuberculosis Treatment}

When a patient abandons the first-line treatment or when it is not effective, it is necessary to use second-line drugs due to the appearance of first-line drug-resistance. Several drugs were developed such as amikacin, capreomycin, moxifloxacin, levofloxacin, linezolid, bedaquilin, cycloserine, ethionamide, clofazimine, kanamycin, $p$-aminosalicylic acid, terizidone, protionamide, and so on. ${ }^{[3]}$ However, the use of a second-line treatment represents some disadvantages compared to the first-line. Second-line drugs require more prolonged use (18-24 months) with many more side effects. The costs and cure rates are also relevant aspects to be taken into consideration: The first-line treatment costs \$ 10-20 per person with a cure rate of more than $95 \%$. On the other hand, the second-line treatment for each patient costs $\$ 19.000$ to 50.000 , with just around $60 \%$ of success. ${ }^{[9]}$

\section{Drug-Resistant Tuberculosis}

One of the major problems in the treatment and cure of tuberculosis is several kinds of drug-resistant tuberculosis. According to World Health Organization, 484,000 people fell ill with drug-resistant tuberculosis in $2018 .^{[4]}$ To standardize the type of treatment, they were basically classified into three types. MDR-TB (multidrug-resistant tuberculosis), XDR-TB (extensive drug resistant or extreme drug resistance), and XXDR or TDR-TB (totally drug-resistance tuberculosis). ${ }^{[12-14]}$ MDR-TB is defined as resistance to, at the minimum, rifampicin, and isoniazid, and it is a common type of resistance. XDR-TB is usually defined as strains resistant to all the current first-line drugs, as well as any fluoroquinolone and, at least, to 1 of 3 injectable second-line drugs (capreomycin, kanamycin or amikacin).${ }^{[12-14]}$ Finally, XXDR or TDR-TB are strains resistant to a wide variety of drugs used to treat tuberculosis. These strains pose a hazard to worldwide public health, once it could make us return to the age of sanatoriums, where there was no adequate treatment and people were isolated dying slowly and painfully. ${ }^{[15]}$

\section{Augmented Reality}

Augmented reality is a technology-based on an interaction between the virtual and real-world, mixing them and expanding their possibilities. Augmented reality calculates the position and angle of the camera image in real-time and adds corresponding images, videos, 3D models. The goal of this technology is to put the virtual world on the screen. The real world interacts, so augmented reality technologies typically include mobile terminals or head-mounted reality systems. ${ }^{[16]}$ This concept is the fundamental difference from virtual reality (VR) that creates virtual environments independent of the real world, making the user immersed and interactive with this new environment. ${ }^{[17,18]}$ In the beginning, both technologies were used and developed in the games industry. Nowadays, they are used in the most various areas of human knowledge. The applications of augmented reality include medical training, auto, and pharmaceutical industry, television broadcast, interior design and modeling, GPS, education, and so on. ${ }^{[19]}$ The use of augmented reality in drug package has great potential for several diseases and could be an indispensable tool, especially in diseases with low medication adherence. ${ }^{[20]}$ For example, in the case of chronic illness, about $50 \%$ of medications are not used according to medical prescription. The treatment non-adherence costs annually billions of dollars around the world. In this context, the U.S. health care system spends per year between $\$ 100-289$ billion. ${ }^{[21]}$

\section{Prescription and Package of Medicines}

The current medication prescription is relatively primitive; in other words, the doctors usually make prescription medicines on their decisions, and on the medical record, they record the frequency and quantity of each drug. When the pharmacy dispenses the medication to the ill, the different medicines are in separate packaging as the doctor's order; there are no instruction on how to use them on the outer package of the medicine. The current packaging uses paper bags and cartons, so it is easy to confuse medicines because the packaging is usually similar. When patients are taking different medications, they withdraw their respective packages carton and various medicines are likely to cause confusion. Note that the appropriate use of each medicine is unclear in this scenario. Besides that, there are patients that are always moving from/to different places due to personal/professional demands. In this case, medicines are taken along this them to leisure or workplaces and it certainly poses a risk, since it is easy for patients to forget the requirements for the appropriate use of each medicine. On the other hand, Brazil has some particular patients with neglected diseases that have some difficulties to understand the prescription due to illiteracy or sight problems 
related or not to old age. All these aspects certainly make the treatment adherence more challenging.

\section{Justification and Objectives}

Tuberculosis treatment suffers from two major problems: the prolonged treatment time (six months) and its severe side effects causing its treatment non-adherence. For example, the percentage of non-adherence in tuberculosis treatment is $15.5 \%$ in Thailand, $24.5 \%$ in South Ethiopia, and $50 \%$ in India. ${ }^{[22,23]}$ After a few weeks using tuberculosis-drugs, the patient's health improves. Due to side effects such as vomiting, headaches, diarrhea, stomach pains, dermatitis, fatigue, and dizziness, the patient does not follow the treatment properly. ${ }^{[24]}$ As a result, there is the development of strains resistant to the medicines used, requiring second-line drugs that have more significant side effects and a long time of use (18-24 months), resulting in much higher costs and less success of cure. Considering these scenarios, we need new strategies to combat non-adherence tuberculosis treatment, or the tragedy will continue to occur. In this context, augmented reality could be an excellent tool in combating non-adherence to the medications against tuberculosis. Using a smartphone in medication packaging could obtain valuable information making the patient be aware of the importance of following the treatment until the end. Besides that, warning signs could also be included in each blister, indicating the exact time to take the medication as well as common questions, psychological help, phone numbers, websites, among other possibilities.

The problem to be solved is to provide an augmented reality based on the medical order medication system to present the dosage of a selected drug of a specific patient in real-time, thereby effectively avoiding patient confusion or mistake.

\section{Results and Discussion}

The present work discusses the proposal of virtual information in the medicine packing to provide information about how to use a particular medication using a quick response (QR) code and augmented reality. This information requires attaching a QR code for executing an augmented reality program to the packing of medicine, giving various pieces of information to a patient through augmented reality aiming the tuberculosis-drugs treatment adherence.

The study suggests the following steps:

(a) Store in a database of medical information including an exterior image of the medicine, description of the disease, posology, dosage, time to take the medicine, alarms, psychological help, websites, phone numbers and other information on a server, and attach a QR code, which enables access to the server and executes the augmented reality program, to the medicine packing;

(b) Take an image of the QR code attached to the medication packaging using a smartphone;

(c) Execute an augmented reality application installed on the smartphone to provide medication packaging sorted by kind on a screen of the smartphone;

(d) Enable a user to select the type of information among the medicine information transmitted to and provided on the screen of the smartphone, accessing to the server through a wireless communication network to download data stored on the server, and implement augmented reality on the screen of the smartphone based on the data by using the augmented reality application.

In step (c), a different augmented reality will be displayed on the smartphone along with a 3D motion image, phrases and/or an audio message in order to provide essential information about the medicine using QR code and augmented reality.
An example of packing and blister of the medicine and corresponding information is shown on the screen of a smartphone in Figure 3.

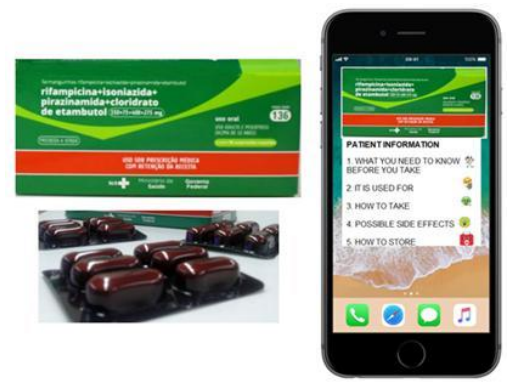

Figure 3 An example of the Brazilian packing and blister used in tuberculosis treatment and the corresponding information on the screen of the smartphone.

In step (a), the augmented reality application is stored in the server. In step (b), the augmented reality application must be downloaded and installed on the smartphone using a wireless connection ( $\mathrm{Wi}-\mathrm{Fi}$ or mobile phone network). After this installation, it will be possible to obtain the information about medicines using the QR code (or similar) printed on the packaging displayed as augmented reality.

Through augmented reality programs, the variety of information provided about the medicine can be updated, only through the update of the app on the smartphone.

\section{Conclusions}

Despite being an old disease, tuberculosis remains a global health problem. It is estimated that one-third of the global population have the Mycobacterium tuberculosis. Therefore, we need new alternatives and strategies to fight against this disease or a tragedy could occur. Currently, a significant problem in the treatment and cure of tuberculosis is patient compliance. As it is a long-term treatment with severe side effects for the patient, the treatment non-adherence is usual and it brings severe consequences for the patient healing, sometimes leading to death. Considering these problems, augmented reality could be an excellent tool to avoid or reduce non-adherence to tuberculosis-drugs, providing several possibilities to help the patient to follow the medical prescription, such as warnings regarding the correct use of the medicines, information about the disease, alarms, psychological help, websites, phone numbers, and so on.

\section{Author Contributions}

All authors contributed equally to the elaboration of the work.

\section{Conflict of Interest}

The authors declare no conflict of interest.

Copyright (c) 2020 Dayse E. de Oliveira, Marcus V. N. de Souza, and Wanise B. G. Barroso. This article is an open access article distributed under the terms and conditions of the Creative Commons Attribution (CC BY) license (http://creativecommons.org/licenses/by/4.0/). The use, distribution or reproduction in other forums is permitted, provided the original author(s) or licensor are credited and that the original publication in this journal is cited, in accordance with accepted academic practice. No use, distribution or reproduction is permitted which does not comply with these terms.

\section{References}

[1] Ziskind, B.; Halioua, B. Tuberculosis in ancient Egypt. Rev. Mal. 
Respir. 2007, 24, 1277-1283.

[2] Daniel, T. M. The History of tuberculosis. Resp. Med. 2006, 100, 1862-1870.

[3] De Souza, M. V. N. Tuberculosis Treatment: In the Search for New Drugs against TB. Bentham ebook, elSBN 2013: 978-1-60805788-7, pp.1-37.

[4] WHO (World Health Organization) www.who.int (accessed in March 2020).

[5] Donoghue, H. D.; Spigelman, M.; Greenblatt, C. L.; Lev-Maor, G.; Bar-Gal, G. K.; Matheson, C.; Vernon, K.; Nerlich, A. G.; Zink, A. R. Tuberculosis: from prehistory to Robert Koch, as revealed by ancient DNA. Lancet Infect. Dis. 2004, 4, 584-592.

[6] Kaufmann, S. H. E.; Schaible, U. E.100th anniversary of Robert Koch's Nobel Prize for the discovery of the tubercle bacillus. Trends in Microbiol. 2005, 13, 469-475.

[7] Kingston, W. Streptomycin, Schatz vs. Waksman, and the balance of credit for discovery; J. Hist. Med. All. Sci. 2004, 59, 441-462.

[8] Souza, M. V. N. Promising Drugs Against Tuberculosis. Recent Pat. on Antiinfect. Drug Discov. 2006, 1, 33-45.

[9] De Souza, M. V. N. Current Status and Future Prospects for New Therapies for Pulmonary Tuberculosis. Curr. Opin. Pulm. Med. 2006, 12, 167-171.

[10] Panchagnula, R.; Agrawal, S.; Ashokraj, Y.; Varma, M.; Sateesh, K.; Bhardwaj, V.; Bedi, S.; Gulati, I.; Parmar, J.; Kaul, C. L.; Blomberg, B.; Fourie, B.; Roscigno, G.; Wire, R.; Laing, R.; Vans, P.; Moore, T. Fixed dose combinations for tuberculosis: Lessons learned from clinical formulation and regulatory perspective. Methods Find. Exp. Clin. Pharmacol. 2004, 26, 703-721.

[11] CDC (Center for Disease Control and Prevention) https://www. cdc.gov/tb/topic/basics/tbhivcoinfection.htm (accessed in March 2020).

[12] Mukherjee, J. S.; Rich, M. L.; Socci, A. R.; Joseph, J. K.; Viru, F. A.; Shi, S. S.; Furin, J. J.; Becerra, M. C.; Barry, D. J.; Kim, J. Y.; Bayona, J.; Farmer, P.; Fawzi, M. C. S.; Seung, K. J. Programs and principles in treatment of multidrug-resistant tuberculosis. Lancet 2004, 363, 474-481.

[13] YuKuo, C.; Wang, W. H.; Huang, C. H.; Chen, Y. H.; Lu, P. L. Resistance to first- and second-line antituberculosis drugs in Southern Taiwan: Implications for empirical treatment J. Micrbiol. Immunol. Infect. 2018, 51, 88-93.

[14] Kendall, E. A.; Cohen, T.; Mitnick, C. D.; Dowdy, D. W. Second line drug susceptibility testing to inform the treatment of rifampinresistant tuberculosis: a quantitative perspective Int. J. Infect. Dis. 2017, 56, 185-189.

[15] Raviglione, M. C.; Smith, I. M. XDR Tuberculosis - Implications for Global Public Health N. Engl. J. Med. 2007, 356-357.

[16] https://www.futurithmic.com/category/augmented-virtual-reality-a ticles/ (accessed in March 2020).

[17] Ventola, C. S. Virtual. Reality in Pharmacy: Opportunities for Clinical, Research, and Educational Applications. Pharm. Ther. 2019, 44, 267-276.

[18] https://www.pharmaceuticalonline.com/doc/harnessing-augmented -reality-in-pharmaceutical-manufacturing-0001 (accessed in March 2020).

[19] https://www.interaction-design.org/literature/article/augmented-real ity-the-past-the-present-and-the-future (accessed in March 2020).

[20] file://C:/Users/User/AppData/Local/Temp/paper10-4.pdf (accessed in March 2020).

[21] Viswanathan, M.; Golin, C. E.; Jones, C. D.; Ashok, M.; Blalock, S. J.; Wines, R. C.; Coker-Schwimmer, E. J.; Rosen, D. L.; Sista, P.; Lohr, K.N. Interventions to improve adherence to self-administered medications for chronic diseases in the united states: a systematic review. Ann. Intern. Med. 2012, 157, 785-795.

[22] Mekonnen, H. S.; Azagew, A. W. Non-adherence to anti-tuberculosis treatment, reasons and associated factors among TB patients attending at Gondar town health centers, Northwest Ethiopia. BMC Res Notes. 2018, 11, 691.

[23] Gube, A. A.; Mengesha, A.; Debalkie, M.; Zeynu, A.; Seid, K.; Shimelis, F.; Bisete, K.; Gebremeskel, K. Assessment of anti-TB drug nonadherence and associated factors among TB patients attending TB clinics in Arba Minch Governmental Health Institutions, Southern Ethiopia. Hidawi 2018, 2018.

[24] Yang, T. W.; Park, H.O.; Jang, H. N.; Yang, J. H.; Kim, S.H.; Moon, S.H.; Byun, J.H.; Lee, C. E.; Kim, J. W.; Kang, D.H. Side effects associated with the treatment of multidrug-resistant tuberculosis at a tuberculosis referral hospital in South Korea: A retrospective study. Medicine (Baltimore) 2017, 96, e7482.

Received April 7, 2020 Accepted April 18, 2020 